\title{
Combined imaginal exposure and cognitive restructuring therapy is more effective than supportive counselling for treating post- traumatic stress disorder
}

Bryant RA, Moulds ML, Guthrie RM, et al. Imaginal exposure alone and imaginal exposure with cognitive restructuring in treatment of posttraumatic stress disorder. J Consult Clin Psychol

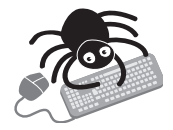

This article contains extra text on the EBMH 2003;71:706-12.

website

For people with post-traumatic stress disorder (PTSD), does cognitive restructuring with prolonged imaginal exposure lead to greater symptom relief than imaginal exposure alone?

METHODS

Design: Randomised controlled trial.
Follow up period: Six months.
$\begin{aligned} & \text { Patients: } 58 \text { people referred to PTSD unit after non-sexual } \\ & \text { assault or traffic accident, displaying PTSD (DSM-IV criteria) } \\ & \text { months. Exclusions: history of psychosis, substance dependence, } \\ & \text { childhood sexual abuse, current suicidal ideation, or aged }<17 \\ & \text { or }>60 \text { years. } \\ & \text { Intervention: Imaginal exposure; imaginal exposure plus } \\ & \text { cognitive restructuring; or supportive counselling for } 8 \text { weekly } 90 \\ & \text { minute sessions with daily homework. } \\ & \text { Outcomes: PTSD symptoms assessed using clinician } \\ & \text { administered PTSD scale. }\end{aligned}$
$\begin{aligned} & \text { Patient follow up: } 78 \% \text {. } \\ & \text { Slinding }\end{aligned}$

Commentary

$\mathrm{R}$ andomised controlled trials have shown that cognitive behaviour therapy is an effective treatment for PTSD. This treatment contains several interventions, including (a) cognitive restructuring (CR) for correcting distorted, self defeating patterns of thinking; (b) live exposure (LE) to distressing but harmless trauma related stimuli; and (c) imaginal exposure (IE) to traumatic memories in order to reduce (habituate) the distress evoked by these memories.

Component studies have sought to determine whether each of these cognitive behavioural interventions contributes to treatment efficacy. If some components are ineffective, then treatment outcome may be improved by concentrating on the more effective interventions. Several studies have sought to determine whether CR contributes to the outcome of cognitive behaviour therapy for PTSD. ${ }^{1-3}$ So far the results have been mixed. Some research suggests that CR improves outcomes, ${ }^{3}$ while other research indicates that outcomes are no better, or possibly worse, when CR is included. ' 2
For correspondence: Dr R A Bryant, School of Psychology, University of New South Wales, Sydney, New South Wales, Australia. r.bryant@unsw.edu.au Sources of funding: The National Health and Medical Research Council.

\section{MAIN RESULTS}

Imaginal exposure with cognitive restructuring significantly reduced PTSD symptoms compared with supportive counselling $(\mathrm{p}<0.05$ at post-treatment and 6 months follow up; see web extra table 1). Imaginal exposure did not significantly reduce PTSD symptoms compared with supportive counselling alone.

\section{CONCLUSIONS}

Imaginal exposure with cognitive restructuring was more effective than supportive counselling for the treatment of PTSD.

This study is the latest component study comparing $I E, C R+I E$, and a supportive counselling control condition. On a number of measures, IE and $C R+I E$ were both more effective than counselling, and $C R+I E$ was somewhat more effective than IE alone, suggesting that CR makes a useful contribution to treatment outcome.

Although this study had a number of methodological strengths, it also has several important limitations that make it difficult to determine generalisability to clinical practice. First, the sample was not representative of treatment-seeking populations because people with sexual abuse were excluded. PTSD commonly arises from sexual abuse. In our recent study of people seeking treatment for PTSD, almost half $(45 \%)$ of the sample had PTSD associated with sexual abuse. ${ }^{4}$ Similarly, other common forms of PTSD, such as combat related PTSD, were not included in the Bryant study.

Bryant's exposure therapy was IE only, which is less effective than LE. ${ }^{5}$ Participants did not receive an audiotape for IE homework, making it more difficult to practice IE homework exercises. With a modestly effective intervention like $\mathrm{IE}$, it would be relatively easy to show that an additional intervention (CR) improves outcome. It is unclear whether a more potent intervention, such as LE, would have been enhanced by the addition of CR. Bryant et al also failed to report whether participants had additional treatment (eg pharmacotherapy) during their pre-to-posttreatment phase or during follow up.

In summary, this study does little to clarify the role of $C R$ in the treatment of PTSD because of their atypical sample and unrepresentative exposure protocol. Indeed, the authors conclude that more research is needed. Although Bryant's study provides further evidence that supportive counselling is not a treatment of choice for PTSD, it provides little other guidance for clinical practice with exposure therapy as it is typically used.

Steven Taylor, PhD

Department of Psychiatry, University of British Columbia, Vancouver, Canada

1 Foa EB, Dancu CV, Hembree EA, et al. A comparison of exposure therapy, stress inoculation training, and their combination for reducing posttraumatic stress disorder in female assault victims. J Consult Clin Psychol 1999;67:194-200.

2 Marks I, Lovell K, Noshirvani H, et al. Treatment of posttraumatic stress disorder by exposure and/or cognitive restructuring. Arch Gen Psychiatry 1998:55:317-25

3 Resick PA, Nishith P, Weaver TL, et al. A comparison of cognitiveprocessing therapy with prolonged exposure and a waiting condition for the treatment of chronic posttraumatic stress disorder in female rape victims. J Consult Clin Psychol 2002;70:867-79.

4 Taylor S, Thordarson DS, Maxfield L, et al. Comparative efficacy, speed, and adverse effects of three PTSD treatments: exposure therapy, EMDR, and relaxation training. J Consult Clin Psychol 2003;71:330-8.

5 Devilly GJ, Foa EB. The investigation of exposure and cognitive therapy: comment on Tarrier et al (1999). J Consult Clin Psychol 2001;69:114-16. 